Polymer Journal, Vol. 4, No. 3, pp 341-349 (1973)

\title{
Fast Reaction and Micro-Brownian Motion of Flexible Polymer Molecules in Solution
}

\author{
Kazuyuki Horie, Itaru Mita, and Hirotaro Kambe \\ Institute of Space and Aeronautical Science, University of \\ Tokyo, Komaba, Meguro-ku, Tokyo, Japan. \\ (Received July 1, 1972)
}

\begin{abstract}
The rate of fast reactions in solution between two flexible polymer molecules with active chain ends is studied in relation to the theory of micro-Brownian motion of polymer segments in solution. The comparison of relaxation times for five types of molecular and segmental motions indicates that the translational diffusion of chain end segments is the rate-determining step. The segmental diffusion coefficient depending on the position based on the model of random flights with correlations is related to the rate constant by using the Smoluchowski equation and the potential energy function for intermolecular interaction. The rate constant can be expressed as a product of three components without any arbitrary parameters,
\end{abstract}

$$
\langle k\rangle=A(\alpha) B(n) C\left(\zeta_{0}, T\right)
$$

where $A(\alpha)$ represets the effect of solution properties, $B(n)$ the effect of molecular weight, and $C\left(\zeta_{0}, T\right)$ the effect of frictional properties of the segment. This equation predicts that the rate constant is inversely proportional to solvent viscosity, decreases with increassing molecular weight to some extent, and is markedly affected by the excluded volume effect and chain flexibility. Close agreement is found between the calculated rate constants and those experimentally obtained.

KEY WORDS Fast Reaction / Polymer Reaction / Diffusion Con-

trol / Rate Constant / Termination / Polymer Radical / Diffusion

Coefficient / Micro-Brownian Motion / Flexibility /

It is well-known that the reaction rate of a fast bimolecular reaction between molecules with highly reactive groups is controlled mainly by the approach process of the two reactants to close proximity. The relation between the rate constant $k$ for bimolecular coagulation and the diffusion coefficient $D$ is given by eq 1 by Smoluchowski, ${ }^{1}$

$$
k=4 \pi R D
$$

where $R$ is the sum of radii of two coagulating species. This relation has been extended to the reaction which includes the electrostatic effects arising from the presence of net charges by Debye ${ }^{2}$ whose equation has been used by Eigen and his coworkers ${ }^{3}$ in their brilliant work on fast ionic reactions in solution.

The fast reaction between two coiling polymer molecules with active chain ends is also supposed to be controlled by the approach pro- cess of the two active sites. One of the good examples of diffusion-controlled polymer reactions is the bimolecular termination step in freeradical polymerization. ${ }^{4}$ In fact, the termination rate constants of two macroradicals are much smaller than those of the recombination of small radicals. The possibility of a diffusion-controlled mechanism for the radical termination in the usual solution polymerization was suggested for the first time by Schulz ${ }^{5}$ in 1956. A great deal of experimental evidence for this assumption has been gathered mainly by North and his coworkers. ${ }^{6,7}$ One of the important points is the inverse proportionality of termination rate constants to solvent viscosity. Reliable molecularweight dependences of bimolecular termination rate constants have been reported by using ESR method $^{8}$ and pulse-radiolysis method. ${ }^{9}$

The theoretical treatment for the diffusioncontrolled termination mechanism has also been 


\section{K. Horie, I. Mita, and H. Kambe}

proposed by several authors..$^{10-18}$ The diffusion process of the reaction can be divided into two relatively independent processes. These are (a) the translational diffusion of the center of mass of the polymer molecules, or macro-Brownian motion of polymer molecules and (b) the translational and rotational diffusion of segments with active chain ends, or micro-Brownian motion of segments. In some models ${ }^{10,13,14}$ these processes are regarded as consecutive and the existence of the "encounter-pair" is assumed while other models ${ }^{11,15-18}$ are concerned with the segmental diffusion of active chain ends in the overlapping space of two interpenetrating polymer molecules. The consensus in both cases is that the translational diffusion process of chain end segments is the rate-determining step for radical termination reactions. The quantitative discussion based on these models, however, was hampered by the lack of an expression for the effective diffusion coefficient for active chain ends in coiling polymers and, therefore, some adjustable parameters have been introduced in all models but those of Burkhart ${ }^{13}$ and Bagdasarian. ${ }^{14}$ Burkhart ${ }^{13}$ has applied the theory of Kuhn and $\mathrm{Kuhn}^{19}$ to the segmental motion of polymer chain ends and proposed for the termination rated constant an expression in terms of solvent viscosity, polymer chain flexibility and chain length. However, this expression predicts an unusual dependence of the termination rate constant on the polymer chain length, caused presumably by the obscure concept of Kuhn and Kuhn's "inner viscosity" of polymer chains. ${ }^{20}$ Bagdasarian $^{14}$ did not use the Smoluchowski relation, but has calculated the mean time needed for the collision of two polymer chain ends in the encounterpair by applying the theory of Frenkel $^{21}$ of the polymer chain relaxation. Though such a model is rather reasonable, his result is unsatisfactory with respect to the molecular-weight dependence of the rate constants. Moreover, there has been no discussion of the effect of excluded volume in a polymer-solvent system on the diffusion controlled rate constants.

The present paper is concerned with a theoretical treatment of the rate of fast reactions in solution between two flexible polymer molecules of equal molecular weights with active chain ends as a function of the distance of the chain end from the molecular center of mass. By using the Snoluchowski equation and the potential energy function, the calculation of rate constants is possible. The effect of coulombic interaction which well be important for the reaction between two charged active sites is ignored here. The resuting expression prodicts the influence of the excluded volume effect and the molecular weight on the rate constant without any arbitrary parameters. Some discussion is carried out on the basic assumptions of the theory and a comparison is made between the calculated rate constants and those experimentally obtained.

\section{RELAXATION TIMES OF VARIOUS MOLECULAR MOTIONS}

A flexible polymer molecule with $N$ monomer units will now be considered. This polymer chain can be divided into $n$ segments of equal length $b$ so that this statistical model of freely jointed segments may have an equal mean-square end-to-end distance $\left\langle h^{2}\right\rangle$ to that of the original molecule. The ratio $N / n$ reflects the chain flexibility of the polymer moelcule and can be expressed approximately by the extent of shortrange interactions along the polymer chain. An approximate value of ten for $N / n$ has been obtained experimentally for vinyl polymers by the fluorescence polarization method. ${ }^{22}$

Relaxation times for five types of molecular motions of reacting molecules and segments should be compared in order to estimate the rate determining step of the reaction.

The relaxation time $\tau_{\mathrm{pc}}$, for the collision of polymer molecules is expressed in eq 2 by using the Smoluchowski equation (eq 1),

$$
\tau_{\mathrm{pc}}=1 / k[\mathrm{C}]=1 / 4 \pi R_{\mathrm{p}} D_{\mathrm{pt}} N_{\mathrm{A}} c
$$

where $k$ is the bimolecular rate constant in $l / \mathrm{mol} \mathrm{sec}, R_{\mathrm{p}}$ is the distance between the centers of mass of two polymer molecules in collision, $D_{\mathrm{p} t}$ is the translational diffusion coefficient of the polymer moecule, and $N_{\mathrm{A}}$ is Avogadro's number. The concentration of polymer molecules is expressed by [C] in $\mathrm{mol} / \mathrm{l}$ and by $c$ $\mathrm{mol} / \mathrm{ml}$. If the Einstein-Stokes relation ${ }^{23}$ between solvent viscosity $\eta_{0}$ and diffusion coefficient of a spherical molecule of radius $a_{\mathrm{p}}$ 
Fast Reaction and Brownian Motion of Polymers

$$
D_{\mathrm{pt}}=k_{\mathrm{B}} T / 6 \pi \eta_{0} a_{\mathrm{p}}
$$

can be used, $\tau_{\mathrm{pc}}$ is given by eq 4 ,

$$
\tau_{\mathrm{pc}}=3 \eta_{0} / 4 c N_{\mathrm{A}} k_{\mathrm{B}} T
$$

where $k_{\mathrm{B}}$ is Boltzmann's constant and $T$ is the absolute temperature. The relaxation time $\tau_{\mathrm{pr}}$ for the rotational diffusion of a spherical polymer molecule is expressed by the Einstein equation, ${ }^{24}$

$$
\tau_{\mathrm{pr}}=4 \pi \eta_{0} a_{\mathrm{p}}{ }^{3} / k_{\mathrm{B}} T
$$

The relaxation time $\tau_{\mathrm{sc}}$ for the collision of chain end segments is given by eq 6 ,

$$
\tau_{\mathrm{sc}}=1 / k[\mathrm{C}]=1 / 4 \pi R_{\mathrm{s}} D_{\mathrm{st}} N_{\mathrm{A}} c
$$

where $R_{\mathrm{S}}$ is the distance between the active sites at the instance of reaction and $D_{\text {st }}$ is the translational diffusion coefficient of the chain end segments. The relaxation time $\tau_{\mathrm{sr}}$ for the rotational diffusion of chain end segment is given by eq 7 in terms of the radius, $a_{\mathrm{s}}$, of the equivalent sphere of the chain end segment.

$$
\tau_{\mathrm{sr}}=4 \pi \eta_{0} a_{\mathrm{s}}^{3} / k_{\mathrm{B}} T
$$

Moreover, it is necessary to estimate the relaxation time $\tau_{\mathrm{po}}$ for the overlapping of two polymer molecules. Graessley ${ }^{25}$ has successfully assumed that the characteristic time for entanglement formation between two molecules approaching each other is of the same order of magnitude as the maximum relaxation time which Rouse $^{26}$ has calculated for the mechanical response of polymer molecules in solution. Thus, it seems reasonable to evaluate $\tau_{\mathrm{po}}$ by using Rouse's equation

$$
\tau_{\text {po }}=6 \eta / \pi^{2} c N_{\mathrm{A}} k_{\mathrm{B}} T
$$

where $\eta$ is the solution viscosity of the system.

In order to compare the orders of magnitude of these relaxation times with experimental values, the calculation has been carried out for the reaction of polymer molecules with $N=10^{4}$ in $1 \mathrm{~g} / 100 \mathrm{~m} l$ solution, by using the values $T=$ $300 \mathrm{~K}, c=10^{-8} \mathrm{~mol} / \mathrm{ml}, \eta_{0}=10^{-2}$ poise, $\eta=10^{-1}$ poise, $a_{\mathrm{p}}=5 \times 10^{-6} \mathrm{~cm}, a_{\mathrm{s}}=10^{-7} \mathrm{~cm}$, and $R_{\mathrm{s}}=$ $3 \times 10^{-8} \mathrm{~cm}$. The results are as follows;

$$
\begin{aligned}
& \tau_{\mathrm{pc}}=3.0 \times 10^{-5} \mathrm{sec} \\
& \tau_{\mathrm{pr}}=3.7 \times 10^{-4} \mathrm{sec} \\
& \tau_{\mathrm{sr}}=3.0 \times 10^{-9} \mathrm{sec}
\end{aligned}
$$

$$
\tau_{\mathrm{po}}=2.4 \times 10^{-4} \mathrm{sec}
$$

A definite numerical value cannot be given for $D_{\text {st }}$. If $D_{\text {st }}$ is supposed to lie in the range of $10^{-6}$ to $10^{-7} \mathrm{~cm}^{2} / \mathrm{sec}$, the range of the value for $\tau_{\mathrm{se}}$ may be given by

$$
\tau_{\mathrm{sc}}=4.4 \times 10^{-4} \sim 4.4 \times 10^{-3} \mathrm{sec}
$$

As the usual experimental rate constant for radical termination $k_{\mathrm{t}}$ has the order of magnitude of $10^{7} \mathrm{l} / \mathrm{mol} \mathrm{sec},{ }^{27}$ the experimental value for overall relaxation time $\tau_{\exp }$ will be given by

$$
\tau_{\text {exp }}=1.0 \times 10^{-2} \mathrm{sec}
$$

Thus the inequality 9 is obtained.

$$
\tau_{\mathrm{sr}} \ll \tau_{\mathrm{pc}}<\tau_{\mathrm{pr}} \text { and } \tau_{\mathrm{po}}<\tau_{\mathrm{sc}}<\tau_{\text {exp }}
$$

As $\tau_{\mathrm{pc}}$ and $\tau_{\mathrm{pr}}$ are very small compared with $\tau_{\text {exp }}$, an equilibrium situation is expected for the macro-Brownian motion of polymer molecules. The overlapping process of polymer molecules may be controlled by the microBrownian rearrangement of polymer segments, essentially the same motion as translational diffusion process of segments. The comparison of the orders of magnitude for $\tau_{\mathrm{po}}$ and $\tau_{\exp }$ indicates that only in a few instances will overlapping contribute to the reaction because the active site is attached to only one segment in a polymer molecule. The process of translational diffusion of chain end segments will be the ratedetermining step, since the rotational rearrangement of monomer units in the segment is sufficiently fast enough for the two active sites to make an encounter during a close contact of two chain and segments. The micro-Brownian motion of chain end segments is supposed to be restricted by surrounding polymeric chains. As $\tau_{\text {sc }}$ based on the $D_{\text {st }}$ values of $10^{-6}$ to $10^{-7}$ $\mathrm{cm}^{2} / \mathrm{sec}$ is smaller than $\tau_{\text {exp }}$, it is adequate to consider that $D_{\text {st }}$ varies markedly according to the position of chain end segment in a polymeric coil. Subsequently, the local rate constant for the collision of chain end segments $k(r)$ will be given on the basis of $D_{\mathrm{st}}(r)$ as a function of the distance $r$ of the chain end from molecular center of mass. The average rate constant $\langle k\rangle$ under the condition of inequality 9 will be obtained through the integration of $k(r)$ multiplied by the probability of finding two thain 
end segments in the same volume element over the whole space. It is supposed that the inequality 9 represents usual cases of free-radical recombination, disproportionation, ionic protonabstracting or other fast reactions between polymer molecules in dilute solution.

\section{DIFFUSION COEFFICIENT OF SEGMENTS}

There seems to be two factors which influence the translational diffusion coefficient of an active chain end in the polymer coil. One of these arises from the fact that the adjacent polymeric chain linked to the chain end segment restricts its mobility. This type of complication is avoided by using the statistical model of equivalent freely jointed segments. The another is caused by the collision of the chain end segment with other segments during the diffusive process. The effect of collisions between segments is expressed by the concentration dependence of the diffusion coefficient, and for polymeric segments a marked dependence will be expected because both of the colliding segments are linked with other segments respectively.

The translational diffusion coefficient of a segment can be expressed by using parameters of microscopic Brownian movements for the segment. By assuming that a collision of a segment with other polymeric segments imposes some restriction on the direction of successive several steps of the segment, we apply to the present case random flight model with correlations according to Tchen. ${ }^{28}$

If the successive steps of a segment are represented by the vectors $\boldsymbol{l}_{i}$, with $\left|\boldsymbol{l}_{i}\right|=\boldsymbol{l}$, then the mean square displacement of a segment per unit time $\left\langle L^{2}\right\rangle$ is given by

$$
\left\langle L^{2}\right\rangle=\left\langle\left(\sum_{i=1}^{\nu} \boldsymbol{l}_{i}\right)^{2}\right\rangle=\sum_{i=1}^{\nu} \sum_{j=1}^{\nu}\left\langle\boldsymbol{l}_{i} \cdot \boldsymbol{l}_{j}\right\rangle
$$

where $\nu$ is the number of steps per unit time. The correlation function between $\boldsymbol{l}_{i}$ and $\boldsymbol{l}_{j}$ is defined by

$$
C_{i, j}=\left\langle\boldsymbol{l}_{i} \cdot \boldsymbol{l}_{j}\right\rangle / \boldsymbol{l}^{2}
$$

and can be considered as a function of $s=$ $|j-i|$ only when the process is stationary. Then

$$
\left\langle L^{2}\right\rangle=2 l^{\nu} \sum_{s=1}^{\nu} \sum_{j=s}^{\nu-1} C_{s}+l^{2} \sum_{j=1}^{\nu} C_{0}
$$

where $C_{0}=1$ and for an uncorrelated case we have

$$
\left\langle L^{2}\right\rangle_{0}=\nu l^{2}
$$

When the fractional volume concentration of segments in the fluid is expressed by $\rho, \nu \rho$ steps in total $\nu$ steps per unit time are colliding steps and may have correlations with successive $s$ steps respectively $\left(C_{s} \neq 0\right.$, for $\left.s=1 \sim s\right)$, while for $\nu(1-\rho)$ stept $C_{s}=0$. Then for this case we have

$$
\left\langle L^{2}\right\rangle=2 l^{2} \rho \sum_{s=1}^{s} \sum_{j=s}^{\nu-1} C_{s}+\nu l^{2}
$$

For a sufficiently large $\nu$, eq 14 becomes to

$$
\left\langle L^{2}\right\rangle=\nu l^{2}\left(1+2 \rho \sum_{s=1}^{s} C_{s}\right)
$$

Because the signs for the $C_{s}$ values are probably negative as shown later, the summation is replaced by $-C$ as

$$
C=-\left(C_{1}+C_{2}+\cdots+C_{s}\right)
$$

then we obtain

$$
\left\langle L^{2}\right\rangle=\nu l^{2}(1-2 C \rho)
$$

As the diffusion coefficient $D$ is related to $\left\langle L^{2}\right\rangle$ by $^{29}$

$$
D=\left\langle L^{2}\right\rangle / 6
$$

The concentration dependence of the diffusion coefficient of a segment can be expressed by

$$
D_{\mathrm{s}}=D_{0}\left(1-2 C_{\rho}\right)
$$

where $D_{0}$ is the diffusion coefficient of the segment when placed separately in the fluid. By using the Einstein relation ${ }^{23}$

$$
D_{0}=k_{\mathrm{B}} T / \zeta_{0}
$$

eq 19 is transformed to

$$
D_{\mathrm{s}}=\left(k_{\mathrm{B}} T / \zeta_{0}\right)(1-2 C \rho)
$$

where $\zeta_{0}$ is the friction coefficient of a segment when placed separately in the fluid.

The fractional volume concentration of segments at the distance $r$ from the molecular center of mass $\rho(r)$ is now calculated on the basis of a Gaussian distribution of segments. The probability $W_{1}(r)$ that we find any segment at the distance $r$ from the molecular center of mass is given by

$$
W_{1}(r)=\left(9 / \pi n b^{2}\right)^{3 / 2} \exp \left(-9 r^{2} / n b^{2}\right)
$$


When each segment is assumed to have a spherical shape, its volume $V_{s}$ is given by

$$
V_{s}=(1 / 6) \pi b^{3}
$$

and we obtain

$$
\rho(r)=n V_{s} W_{1}(r)=\left(9 / 2 \pi^{1 / 2} n^{1 / 2}\right) \exp \left(-9 r^{2} / n b^{2}\right)
$$

Now we introduce an assumption that the diffusion coefficient of the segment (relative to the coordinate) at the molecular center of mass $D_{s}(r=0)$ is equal to the translational diffusion coefficient of polymer molecule $D_{\mathrm{pt}}$. The validity of this assumption will be discussed in the final section below. According to Kirkwood and Riseman, ${ }^{30} D_{\mathrm{pt}}$ is given by

$$
D_{\mathrm{pt}}=\left(k_{\mathrm{B}} T / n \zeta_{0}\right)\left(1+8 \lambda_{0} n^{1 / 2} / 3\right)
$$

where

$$
\lambda_{0}=\zeta_{0} / 6^{1 / 2} \pi^{3 / 2} \eta_{0} b
$$

The from eq 21 and 25 , we obtain

$$
C=\{1 / 2 \rho(0)\}\left\{1-\left(1+8 \lambda_{0} n^{1 / 2} / 3\right) / n\right\}
$$

Consequently, the diffusion coefficient of a segment at the distance $r$ from the center of mass of the polymer molecule composed of $n$ segments is expressed in

$$
\begin{aligned}
D_{\mathrm{s}}(r) & =\left(k_{\mathrm{B}} T / \zeta_{0}\right)[1-\{1-(1 \\
& \left.\left.\left.+8 \lambda_{0} n^{1 / 2} / 3\right) / n\right\} \exp \left(-9 r^{2} / n b^{2}\right)\right]
\end{aligned}
$$

\section{RATE CONSTANT FOR POLYMER REACTIONS}

The reaction rate constant $k$ is expressed by eq 29 in terms of the diffusion coefficient $D$ and the rate constant for the fast chemical process $k_{\mathrm{c}}$, according to the modified Smoluchowski equation $^{31}$

$$
k=4 \pi R D /\left(1+4 \pi R D / k_{\mathrm{c}}\right)
$$

The original Smolchowski equation 1 corresponds to the extreme case when the chemical process succeeding to the diffusion process proceeds much faster than the latter; i.e.,

$$
k_{\mathrm{c}} \gg 4 \pi R D
$$

As the diffusion coefficient $D$ is the sum of segmental diffusion coefficients in $\mathrm{cm}^{2} / \mathrm{sec}$ for each reactant, the rate constant at point $R$ in $l / \mathrm{mol} \mathrm{sec}$ can be given by eq 31 for the condi- tion of inequality given in eq 30 ,

$$
k_{\mathrm{R}}\left(r_{\mathrm{A}}, r_{\mathrm{B}}\right)=4 \pi R_{\mathrm{s}}\left\{D_{\mathrm{s}}\left(r_{\mathrm{A}}\right)+D_{\mathrm{s}}\left(r_{\mathrm{B}}\right)\right\} N_{\mathrm{A}} \times 10^{-3}
$$

where $r_{A}$ and $r_{B}$ are the distance of point $R$ from the centers of mass of molecules $A$ and $\mathrm{B}$, respectively, and $\boldsymbol{R}_{\mathrm{s}}$ is expressed in $\mathrm{cm}$.

As pointed out already, $\tau_{\mathrm{po}}$ is much smaller than $\tau_{\text {exp }}$. Consequently it is permisible to consider that polymer molecules are thermodynamically in equilibrium in solution before the termination reaction takes place. Then, the rate constant averaged over the whole space $\langle k\rangle$ will be given by the integration of $k_{\mathrm{R}}\left(r_{\mathrm{A}}, r_{\mathrm{B}}\right)$ multiplied by the probability that two reacting chain end segments lie in a given point $R$ in the equilibrium condition as

$$
\begin{aligned}
& \langle k\rangle=\iint k_{\mathrm{R}}\left(r_{\mathrm{A}}, r_{\mathrm{B}}\right) \\
& \times \exp \left(-\Delta F(a) / k_{\mathrm{B}} T\right) W_{2}\left(r_{\mathrm{A}}\right) W_{2}\left(r_{\mathrm{B}}\right) 4 \pi r_{\mathrm{A}}{ }^{2} 4 \pi{r_{\mathrm{B}}}^{2} \mathrm{~d} r_{\mathrm{B}} \mathrm{d} r_{\mathrm{A}}
\end{aligned}
$$

where $\Delta F(a)$ is the potential energy of interaction of two molecules, while $a$ is the distance between their centers of mass. $W_{2}(r)$ is the probability of finding the chain end segment at the distance $r$ from the molecular center of mass.

It is to be noted that the excluded-volume effect which is important in the theory of solution properties is introduced through $\Delta F(a)$ and $W_{2}(r)$. The intermolecular interaction $\Delta F(a)$ is expressed $\mathrm{as}^{32}$

$$
\Delta F(a)=k_{\mathrm{B}} T \beta n^{2}\left(9 / 2 \pi n \alpha^{2} b^{2}\right)^{3 / 2} \exp \left(-9 a^{2} / 2 n \alpha^{2} b^{2}\right)
$$

where $\alpha$ is the expansion factor and the effective excluded volume of the segment $\beta$ is given by eq 34 in terms of Flory's "ideal" temperature $\theta$,

$$
\beta=\beta_{0}(1-\theta / T)
$$

The probability $W_{2}(r)$ is given by

$$
W_{2}(r)=\left(9 / 2 \pi n \alpha^{2} b^{2}\right)^{3 / 2} \exp \left(-9 r^{2} / 2 n \alpha^{2} b^{2}\right)
$$

In the coordinate system shown in Figure 1, we have

$$
r_{\mathrm{A}}^{2}+{r_{\mathrm{B}}}^{2}=2 q^{2}+a^{2} / 2
$$

Equation 32 substituted by eq 35 is expressed in eq 37 as a function of $q$ and $a$, 


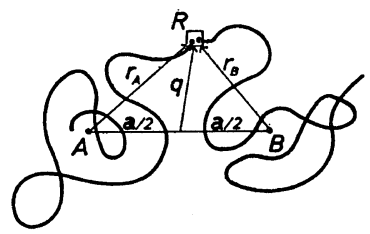

Figure 1. Coordinate system of two Gaussian chains at the time of reaction.

$$
\begin{aligned}
\langle k\rangle \simeq & \left(9 / 4 \pi n \alpha^{2} b^{2}\right)^{3 / 2} \int_{0}^{\infty} \exp \left(-\Delta F(a) / k_{\mathrm{B}} T\right) \\
& \times \exp \left(-9 a^{2} / 4 n \alpha^{2} b^{2}\right) 4 \pi a^{2} \mathrm{~d} a^{2} \\
& \times\left(9 / \pi n \alpha^{2} b^{2}\right)^{3 / 2} \int_{0}^{\infty} k_{\mathrm{R}}(q) \\
& \times \exp \left(-9 q^{2} / n \alpha^{2} b^{2}\right) 4 \pi q^{2} \mathrm{~d} q
\end{aligned}
$$

where an approximation

$$
k_{\mathrm{R}}\left(r_{\mathrm{A}}, r_{\mathrm{B}}\right)=k_{\mathrm{R}}(q)=8 \pi R_{\mathrm{s}} N_{\mathrm{A}} D_{\mathrm{s}}(q) \times 10^{-3}
$$

is used. By introducing new parameters $x, y$, ane the interaction parameter $z$ as

$$
\begin{gathered}
x^{2}=a^{2} / n \alpha^{2} b^{2}, \quad y^{2}=q^{2} / n \alpha^{2} b^{2} \\
z=\left(3 / 2 \pi b^{2}\right)^{3 / 2} \beta n^{1 / 2}
\end{gathered}
$$

and by substituting eq 28,33 , and 38 into eq 37 , we obtain

$$
\begin{aligned}
\langle k\rangle= & \left(27 / 2 \pi^{1 / 2}\right) \int_{0}^{\infty} \exp \left\{-3^{3 / 2} z \alpha^{-3}\right. \\
& \left.\times \exp \left(-9 x^{2} / 2\right)\right\} x^{2} \exp \left(-9 x^{2} / 4\right) \mathrm{d} x \\
& \times\left(108 / \pi^{1 / 2}\right)\left(8 \times 10^{-3} \pi R_{\mathrm{S}} N_{\mathrm{A}} k_{\mathrm{B}} T / \zeta_{0}\right) \\
& \times \int_{0}^{\infty}\left[1-\left\{1-\left(1+8 \lambda_{0} n^{1 / 2} / 3\right) / n\right\}\right. \\
& \left.\times \exp \left(-9 y^{2}\right)\right] y^{2} \exp \left(-9 y^{2}\right) \mathrm{d} y
\end{aligned}
$$

The frictional coefficient of a segment $\zeta_{0}$ is assumed to be equal to the friction coefficient of a monomeric unit multiplied by the number of monomeric units in a segment $N_{0}$, and will be given by

$$
\zeta_{0}=6 \pi \eta_{0} a_{\mathrm{m}} N_{0}
$$

where $a_{\mathrm{m}}$ is the hydrodynamic radius of a monomeric unit. The values of $N_{0}$ may approximately be determined by eq 43 ,

$$
N_{0}=N / n=\left\langle h^{2}\right\rangle /\left\langle h^{2}\right\rangle_{\text {of } \mathrm{j}}
$$

where $\left\langle h^{2}\right\rangle_{0 \mathrm{fj}}$ is the mean-square end-to-end distance for freely jointed monomeric units.
Thus, we obtain finally the rate equation for the bimolecular fast reaction of polymers with active chain ends as a product of three components, each representing the effect of solution properties $(A)$, molecular weight $(B)$, and frictional properties $(C)$, respectively,

$$
\langle k\rangle=A(\alpha) B(n) C\left(\zeta_{0}, T\right)
$$

where

$$
\begin{aligned}
& A(\alpha)=\left(27 / 2 \pi^{1 / 2}\right) \int_{0}^{\infty} \exp \left\{-27 z \alpha^{-3} .\right. \\
&\left.\times \exp \left(-9 x^{2} / 2\right)\right\} x^{2} \exp \left(-9 x^{2} / 4\right) \mathrm{d} x \\
& B(n)=\left(108 / \pi^{1 / 2}\right) \\
& \times \int_{0}^{\infty}\left[1-\left\{1-\left(1+8 \lambda_{0} n^{1 / 2} / 3\right) / n\right\}\right. \\
&\left.\times \exp \left(-9 y^{2}\right)\right] y^{2} \exp \left(-9 y^{2}\right) \mathrm{d} y \\
& C\left(\zeta_{0}, T\right)=4 \times 10^{-3} R_{\mathrm{S}} N_{\mathrm{A}} k_{\mathrm{B}} T / 3 N_{0} \eta_{0} a_{\mathrm{m}}
\end{aligned}
$$

When the active site is situated on a randomly selected position along the polymer chain, we must use $W_{1}$ modified by $\alpha$ instead of $W_{2}$ in eq 32 , and finally the rate constant for this case $\langle k\rangle^{\prime}$ is given by

$$
\langle k\rangle^{\prime}=A^{\prime}(\alpha) B^{\prime}(n) C\left(\zeta_{0}, T\right)
$$

where

$$
\begin{aligned}
A^{\prime}(\alpha)= & \left(27 \sqrt{2} / \pi^{1 / 2}\right) \int_{0}^{\infty} \exp \left\{-27 z \alpha^{-3}\right. \\
& \left.\times \exp \left(-9 x^{2} / 2\right)\right\} x^{2} \exp \left(-9 x^{2} / 2\right) \mathrm{d} x \\
B^{\prime}(n)= & \left(216 \sqrt{2} / \pi^{1 / 2}\right) \\
& \times \int_{0}^{\infty}\left[1-\left\{1-\left(1+8 \lambda_{0} n^{1 / 2} / 3\right) / n\right\}\right. \\
& \left.\times \exp \left(-9 y^{2}\right)\right] y^{2} \exp \left(-18 y^{2}\right) \mathrm{d} y
\end{aligned}
$$

The factors influenced by the excluded volume effect $A(\alpha)$ and $A^{\prime}(\alpha)$ are calculated by using the Flory-Fisk equation ${ }^{33}$

$$
\alpha^{5}-\alpha^{3}=0.648 z\left\{1+0.969\left(1+z / \alpha^{3}\right)^{-2 / 3}\right\}
$$

They are shown in Figure 2 for the usual range of $\alpha$ values. The factors directly affected by molecular weight $B(n)$ and $B^{\prime}(n)$ are also calculated and shown in Figure 3 for the usual range of $n$. In order to simplify the numerical calculation, $\lambda_{0}$ is approximated by $\left.(3 / 2 \pi)\right)^{1 / 2}$ The effect of the molecular weight also appears inplicitly in $A(\alpha)$ and $A^{\prime}(\alpha)$, because $\alpha$ increases with increasing $n$ when $\alpha$ is larger than unity. 


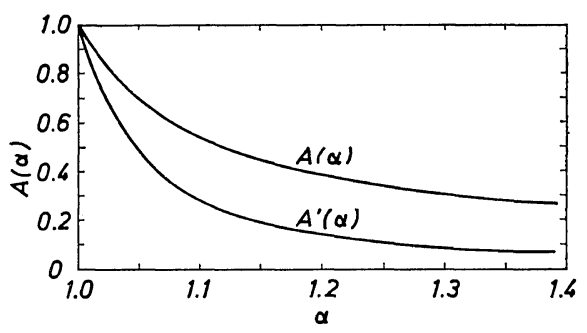

Figure 2. Calculated values of $A(\alpha)$ and $A^{\prime}(\alpha)$ depending on the expansion factor $\alpha$.

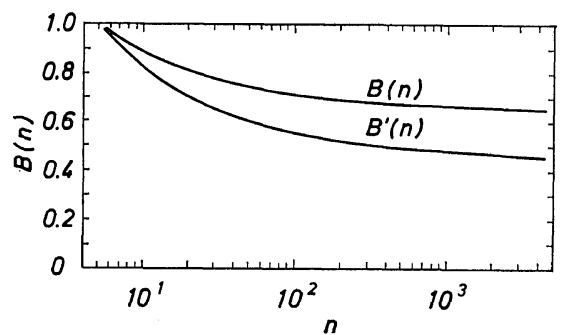

Figure 3. Calculated values of $B(n)$ and $B^{\prime}(n)$ depending of segments in a polymer molecule $n$.

Equation 47 indicates the inverse proportionality of the rate constant to solvent viscosity. But it is noted that the change of solvent affects the rate constant not only through the change in viscosity but also through the change in thermodynamic properties of the solution. Experimental inverse proportionality of the rate constant to the solvent viscosity using various solvents will be guaranteed only when their solvent powers are similar. The rate constant in good solvents is nearly proportional to the inverse half power of molecular weight when $n$ is not so large and becomes independent of it for large $n$. The effect of chain flexibility will be expressed by the change of $N_{0}$ in eq 43 .

\section{DISCUSSION}

The Smoluchowski equation (eq 1) has been derived by means of equation for diffusion under a concentration gradient. However there is a disturbing aspect concerning the nature of the concentration gradient, as in solution the collision is actually caused by the Brownian motion of particles regardless of the concentration gradient. This problem has been discussed by Collins and Kimball, ${ }^{31}$ who have ascertained the fundamental validity of the Smoluchowski equation by using a physically different model.

The discussion has already been given above in RELAXATION TIMES OF VARIOUS MOLECULAR MOTIONS concerning the applicability of the assumption that an equilibrium exists for the motions of polymer molecules including the overlapping process. The fact may be noted here that Frenkel ${ }^{21}$ has evaluated the relaxation time of polymer chain rearrangement in a quite different manner to be the same order of magnitude as $\tau_{\mathrm{p} 0}$ in eq 8 .

Some comment should be made concerning the correlation parameter $C$, which plays a role in the deviation of the segmental diffusion coefficient $D_{s}(r)$ depending on distance $r$. According to the postulated model, the reflection of a diffusing segment by collision is the main reason of correlation between successive steps. So $C_{s}$ $(s=1 \sim s)$ will have negative values between zero and minus unity and rapidly approach zero with increasing $s$. The values of $C=-\sum_{s=1}^{s} C_{s}$ can be calculated according to eq 27 as a function of the number of monomer units in a molecule $N$, and one obtains $C=1.0$ for $N=5 \times 10^{2}, C=1.6$ for $N=5 \times 10^{3}$. These values are thought to be reasonable in spite of some assumptions during the course of the derivation. The chain entanglement supposed for polymer molecules of high molecular weight also causes the relatively large values of $C$ for large $N$. As it is difficult to evaluate $D_{s}(r=0)$ precisely, we have assumed that $D_{s}(r=0)$ is equal to $D_{\mathrm{pt}}$. It should be noted that even if it deviated from $D_{\mathrm{pt}}$, the resulting value of $B(n)$ would not deviate appreciably from the values shown in Figure 3 .

The reported values of termination rate constants in usual radical polymerizations show wide scatter in the range of $2 \times 10^{6}$ to $7 \times 10^{7}$ $l / \mathrm{mol} \mathrm{sec},{ }^{27}$ and cannot be used for the precise verification of eq $44 \sim 47$. Borgwardt, Schnabel and Henglein ${ }^{9}$ have directly measured rate constants for the recombination of poly(oxyethylene) radicals in aqueous solution generated by the pulse-radiolysis method. The numerical evaluation of eqs $44 \sim 47$ and eqs $47 \sim 50$, therefore, is performed by assuming that the same conditions prevail as did during their experiments.

The limiting viscosity number $[\eta]$ of poly(oxy- 
K. Horie, I. Mita, and H. Kambe

Table I. Calculation of rate constants $\langle k\rangle$ and $\langle k\rangle^{\prime}$ according to eq $44 \sim 47$ and eq $47 \sim 50$

\begin{tabular}{|c|c|c|c|c|c|c|c|}
\hline Polyoxyethylene & $N$ & $\alpha$ & $A(\alpha)$ & $B(n)$ & $\begin{array}{r}\langle k\rangle_{\text {calc }} \\
l / \mathrm{mol} \mathrm{sec}\end{array}$ & $\begin{array}{l}\langle k\rangle^{\prime} \text { calc, } \\
l / \mathrm{mol} \mathrm{sec}\end{array}$ & $\begin{array}{l}\langle k\rangle_{\text {exp }}{ }^{\mathrm{a}} \\
l / \mathrm{mol} \mathrm{sec}^{2}\end{array}$ \\
\hline \multirow{6}{*}{$\begin{array}{l}\text { Polyoxyethylene } \\
\text { in water }\end{array}$} & 11 & & & & & & $4.6 \times 10^{8}$ \\
\hline & 38 & 1.10 & 0.55 & 0.90 & $6.1 \times 10^{8}$ & $2.9 \times 10^{8}$ & $2.2 \times 10^{8}$ \\
\hline & 118 & 1.14 & 0.46 & 0.77 & $4.3 \times 10^{8}$ & $1.6 \times 10^{8}$ & $1.2 \times 10^{8}$ \\
\hline & 409 & 1.20 & 0.39 & 0.71 & $3.4 \times 10^{8}$ & $9.7 \times 10^{8}$ & $8.0 \times 10^{8}$ \\
\hline & 775 & 1.24 & 0.36 & 0.69 & $3.1 \times 10^{8}$ & $7.8 \times 10^{7}$ & $6.8 \times 10^{7}$ \\
\hline & 3000 & 1.34 & 0.30 & 0.67 & $2.5 \times 10^{8}$ & $4.8 \times 10^{7}$ & $5.6 \times 10^{7}$ \\
\hline \multirow{3}{*}{$\begin{array}{l}\text { Polystyrene } \\
\text { in toluene }\end{array}$} & $10^{2}$ & 1.14 & 0.46 & 0.90 & $1.3 \times 10^{8}$ & & \\
\hline & $10^{3}$ & 1.28 & 0.33 & 0.71 & $7.5 \times 10^{7}$ & & \\
\hline & $10^{4}$ & 1.48 & 0.25 & 0.67 & $5.3 \times 10^{7}$ & & \\
\hline
\end{tabular}

a Data with pulse-radiolysis method. ${ }^{9}$

ethylene) in water is related to the molecular weight $M$. by ${ }^{34}[\eta]=6.4 \times 10^{-5} M^{0.82}$. By inserting eq 52 in the familiar relationship ${ }^{35}$

$$
[\eta] / M^{1 / 2}=\Phi\left(\left\langle h^{2}\right\rangle_{0} / M\right)^{3 / 2} \alpha^{3}
$$

with the parameter $\Phi=2.5 \times 10^{21}$ and $\left\langle h^{2}\right\rangle_{0} / M=$ $0.60 \times 10^{-16} \mathrm{~cm}^{2},{ }^{36}$ we find $\alpha=1.34$ for a polymer of $N=3 \times 10^{3}$. The values of $\alpha$ for lower $N$ are estimated by considering the square-root proportionality of $z$ to $N$ and eq 51. The recombination rate constants $\langle k\rangle$ and $\langle k\rangle^{\prime}$ for the polymer of $N$ monomer units can be calculated by using eq $44 \sim 47$ and eq $47 \sim 50$, respectively, with the values of $N_{0}=4.1,{ }^{36} a_{\mathrm{m}}=2.2 \times 10^{-8} \mathrm{~cm}$, $R_{s}=3 \times 10^{-8} \mathrm{~cm},{ }^{10,37} T=298 \mathrm{~K}$ and $\eta_{0}=0.89 \times 10^{-2}$ poise.

The calculated and experimentally found values of $\langle k\rangle$ are summarized in Table I together with the values of $A(\alpha)$ and $B(n)$ obtained in the course of calculation. The dependences of $\langle k\rangle_{\text {calc }},\langle k\rangle_{\text {calc }}^{\prime}$ and $\langle k\rangle_{\text {exp }}$ on the number of monomer units $N$ for polyoxyethylene are visualized in Figure 4. It can be noted that the $\langle k\rangle^{\prime}$ values calculated for the case of random distribution of active sites are in close agreement with the experimental values, if we take into consideration that there are no adjustable parameters in eq $47 \sim 50$. Supposedly then active sites in polyoxyethylene are randomly produced along polymer chains by the attack of hydroxy radicals in the case of pulse-radiolysis method. The calculated rate constants for the termination reaction of polystyrene of $N=10^{2}, 10^{3}$, and $10^{4}$ in toluene at $285 \mathrm{~K}$ with the appropriate values of $\alpha^{38}$ are also indicated in Table $\mathrm{I}$.

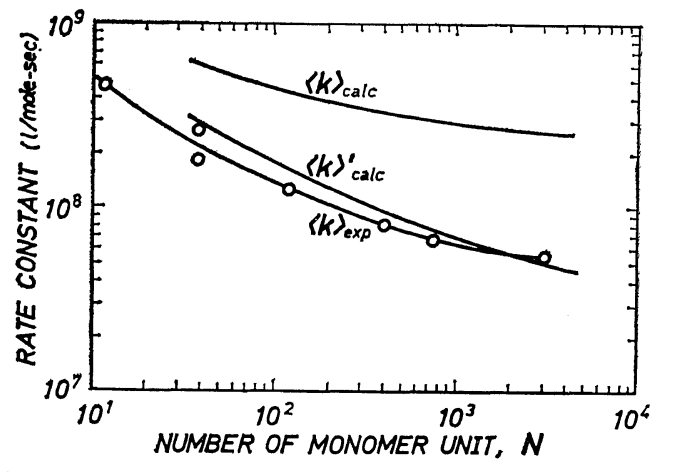

Figure 4. Comparison of calculated rate constants $\langle k\rangle_{\text {calc }}$ and $\langle k\rangle^{\prime}$ calc for the recombination reaction of polyoxyethylene radicals in water with experimental values $\langle k\rangle_{\text {exp }}$.

The molecular weight dependehce of the diffusion-controlled rate constant expressed in eq $44 \sim 47$ seems to be qualitatively similar to that given by Benson and North. ${ }^{10}$ However, they have introduced in their equation correction parameters $\boldsymbol{P}_{\mathrm{AB}}$ and $N_{\mathrm{L}}$, whereas all parameters in our equations $44 \sim 47$ are given precisely by a study of the solution properties of polymer molecules. In usual radical polymerizations, the molecular weight distribution expressed by the ratio $\bar{M}_{w} / \bar{M}_{n}$ lies between 1.5 and 2.0 , and the reported molecular weight independence of the termination rate constant for methyl methacrylate polymerization ${ }^{37}$ is not necessarily reliable because of the polydispersity of the reacting polymer chains. For the direct verification of eq $44 \sim 47$ it is necessary to measure rate constants for the fast bimolecular reaction of 
monodisperse polymers with active chain ends. This kind of work is now being conducted in our laboratory.

It is concluded from the above that the rate constant of the fast reaction in solution between monodisperse polymer molecules with active chain ends can be expressed by eq $44 \sim 47$ without any adjustable parameter. The rate constant is inversely proportional to solvent viscosity, and decreases with increasing molecular weight to some extent. The rate constant is markedly affected by the excluded volume effect of the polymer-solvent system. Most of free-radical recombination and disproportionation, ionic proton-abstracting or other fast reactions between polymer molecules will be included in this type of diffusion-controlled reaction.

Acknowledgments. The authors express their thanks to Dr. Y. Takano of Gunma University, Prof. H. H. G. Jellinek of Clarkson College of Technology, and Dr. K. Ito of Government Industrial Research Institute of Nagoya for their kind discussion. One of the authors (K.H.) is grateful to SAKKOKAI Foundation for a grant.

\section{REFERENCES}

1. M. Smoluchowski, Z. Phys. Chem., 92, 129 (1918).

2. P. Debye, Trans. Electrochem. Soc., 82, 265 (1942).

3. M. Eigen, Angew. Chem., 75, 489 (1963).

4. K. Horie, I. Mita, and H. Kambe, J. Polym. Sci. Part A-1, 7, 2561 (1969).

5. G. V. Schulz, Z. Phys. Chem. N. F., 8, 284 (1956).

6. A. M. North, "Progress in High Polymers" Vol. 2, J. C. Robb and F. W. Peaker, Ed., Iliffe, London, 1968, p 95.

7. K. Yokota, Kobunshi, (High Polymers) 19, 566 (1970).

8. H. Fischer, Makromol. Chem., 98, 197 (1966).

9. U. Borgwardt, W. Schnabel, and A. Henglein, Makromol. Chem., 127, 176 (1969).

10. S. W. Benson and A. M. North, J. Amer. Chem. Soc, 84, 935 (1962).

11. P. E. M. Allen and C.R. Patrick, Makromol.
Chem., 72, 106 (1964).

12. A. M. North, Makromol. Chem., 83, 15 (1965).

13. R. D. Burkhart, J. Polym. Sci. Part A, 3, 883 (1965).

14. Kh. S. Bagdasarian, Vysokomol. Soedin. Ser. B, 9, 10 (1967).

15. A. F. Morini and G. V. Schulz, Makromol. Chem., 118, 313 (1969).

16. K. Ito, J. Polym. Sci. Part A-1, 8, 1823 (1970).

17. S. V. Kozlov, A. A. Ovchinnikov, and N. S. Enikolopian, Vysokomol. Soedin. Ser. A, 12, 987 (1970).

18. O. F. Olaj, Makromol. Chem., 147, 235 (1971).

19. W. Kuhn and H. Kuhn, Helv. Chim. Acta, 28, 1533 (1945).

20. N. Saito, "Introduction to Polymer Physics" (Revised ed.), Syokabo, Tokyo, 1967, p 328.

21. J. Frenkel, "Kinetic Theory of Liquids," Dover, New York, N. Y., 1955, p 462.

22. Y. Nishijima, $\dot{A}$. Teramoto, and S. Hiratsuka, J. Polym. Sci., Part A-2, 5, 23 (1967).

23. A. Einstein, Ann. Phys., 17, 549 (1905).

24. A. Einstein, Ann. Phys., 19, 371 (1906).

25. W. W. Graessley, J. Chem. Phys., 43, 2696 (1965).

26. P. E. Rouse, Jr. J. Chem. Phys., 21, 1272 (1953).

27. C. H. Bamford, W. G. Barb, A. D. Jenkins, and P. F. Onyon, "The Kinetics of Vinyl Polymerization by Radical Mechanisms," Butterworths, London, 1958, p 55.

28. C. M. Tchen, J. Chem. Phys., 20, 214 (1952).

29. S. Chandrasekhar, Rev. Modern Phys., 15, 1 (1943).

30. J. G. Kirkwood and J. Riseman, J. Amer. Chem. Soc., 16, 565 (1948).

31. F. C. Collins and G. E. Kimball, J. Colloid Sci., 4, 425 (1949).

32. P. J. Flory and W. R. Krigbaum, J. Chem. Phys., 18, 1086 (1950).

33. P. J. Flory and S. Fisk, J. Chem. Phys., 44, 2243 (1966).

34. F. E. Bailey and R. W. Callard, J. Appl. Polym. Sci., 1, 56 (1959).

35. P. J. Flory and T. G. Fox, J. Amer. Chem., Soc., 73, 1904 (1951).

36. J. E. Mark and P. J. Flory, J. Amer. Chem. Soc., 87, 1415 (1965).

37. G. V. Schulz and J. P. Fischer, Makromol. Chem., 107, 253 (1965).

38. G. C. Berry, J. Chem. Phys., 44, 4550 (1966). 